Bull. Korean Math. Soc. 49 (2012), No. 3, pp. 573-580

http://dx.doi.org/10.4134/BKMS.2012.49.3.573

\title{
$(1, \lambda)$-EMBEDDED GRAPHS AND THE ACYCLIC EDGE CHOOSABILITY
}

\author{
Xin Zhang, Guizhen Liu, and Jian-Liang Wu
}

\begin{abstract}
A $(1, \lambda)$-embedded graph is a graph that can be embedded on a surface with Euler characteristic $\lambda$ so that each edge is crossed by at most one other edge. A graph $G$ is called $\alpha$-linear if there exists an integral constant $\beta$ such that $e\left(G^{\prime}\right) \leq \alpha v\left(G^{\prime}\right)+\beta$ for each $G^{\prime} \subseteq G$. In this paper, it is shown that every $(1, \bar{\lambda})$-embedded graph $G$ is 4 -linear for all possible $\lambda$, and is acyclicly edge- $(3 \Delta(G)+70)$-choosable for $\lambda=1,2$.
\end{abstract}

\section{Introduction and basic definitions}

In this paper, all graphs considered are finite, simple and undirected. We use $V(G), E(G), \delta(G)$ and $\Delta(G)$ to denote the vertex set, the edge set, the minimum degree and the maximum degree of a graph $G$, respectively. Let $e(G)=|E(G)|$ and $v(G)=|V(G)|$. Moreover, for an embedded graph $G$ (i.e., a graph that can be embedded on a surface), by $F(G)$ we denote the face set of $G$. Let $f(G)=|F(G)|$. The girth $g(G)$ of a graph $G$ is the length of the shortest cycle of $G$. A $k$-, $k^{+}$- and $k^{-}$-vertex (or face) is a vertex (or face) of degree $k$, at least $k$ and at most $k$, respectively. A graph $G$ is called $\alpha$-linear if there exists an integral constant $\beta$ such that $e\left(G^{\prime}\right) \leq \alpha v\left(G^{\prime}\right)+\beta$ for each $G^{\prime} \subseteq G$. Furthermore, if $\beta \geq 0$, then $G$ is said to be $\alpha$-nonnegative-linear; and if $\beta<0$, then $G$ is said to be $\alpha$-negative-linear. For other undefined concepts we refer the reader to [3].

A mapping $c$ from $E(G)$ to the sets of colors $\{1, \ldots, k\}$ is called a proper edge- $k$-coloring of $G$ provided that any two adjacent edges receive different colors. A proper edge- $k$-coloring $c$ of $G$ is called an acyclic edge- $k$-coloring of $G$ if there are no bichromatic cycles in $G$ under the coloring $c$. The smallest number of colors such that $G$ has an acyclic edge coloring is called the acyclic

Received January 31, 2011.

2010 Mathematics Subject Classification. 05C10, 05C15.

Key words and phrases. ( $1, \lambda$ )-embedded graph, $\alpha$-linear graph, acyclic edge choosability.

This research is partially supported by Graduate Independent Innovation Foundation of Shandong University (No. yzc10040) and National Natural Science Foundation of China (No. 10971121, 11026184, 61070230).

The first author is under the support from the Chinese Ministry of Education Prize for Academic Doctoral Fellows. 
edge chromatic number of $G$, denoted by $\chi_{a}^{\prime}(G)$. A graph is said to be acyclic edge- $f$-choosable, whenever we give a list $L_{e}$ of $f(e)$ colors to each edge $e \in$ $E(G)$, there exists an acyclic edge- $k$-coloring of $G$, where each element is colored with a color from its own list. If $\left|L_{e}\right|=k$ for edge $e \in E(G)$, we say that $G$ is acyclicly edge- $k$-choosable. The minimum integer $k$ such that $G$ is acyclicly edge- $k$-choosable is called the acyclic edge choice number of $G$, denoted by $\chi_{c}^{\prime}(G)$.

Acyclic coloring problem introduced in [8] has been extensively studied in many papers. One of the famous conjectures on the acyclic edge chromatic number is due to Alon, Sudakov and Zaks [2]. They conjectured that $\chi_{a}^{\prime}(G) \leq$ $\Delta(G)+2$ for any graph $G$. Alon et al. [1] proved that $\chi_{a}^{\prime}(G) \leq 64 \Delta(G)$ for any graph $G$ by using probabilistic arguments. This bound for arbitrary graph was later improved to $16 \Delta(G)$ by Molloy and Reed [9] and recently improved to $9.62 \Delta(G)$ by Ndreca et al. [10]. In 2008, Fiedorowicz et al. [7] proved that $\chi_{a}^{\prime}(G) \leq 2 \Delta(G)+29$ for each planar graph $G$ by applying a combinatorial method. Nowadays, acyclic coloring problem has attracted more and more attention since Coleman et al. [4, 5] identified acyclic coloring as the model for computing a Hessian matrix via a substitution method. Thus to consider the acyclic coloring problems on some other special classes of graphs seems interesting.

A graph is called 1-planar if it can be drawn on the plane so that each edge is crossed by at most one other edge. The notion of 1-planar graph was introduced by Ringel [11] while trying to simultaneously color the vertices and faces of a planar graph such that any pair of adjacent/incident elements receive different colors. In fact, from a planar graph $G$, we can construct a 1-planar graph $G^{\prime}$ with its vertex set being $V(G) \cup F(G)$, and any two vertices of $G^{\prime}$ being adjacent if and only if their corresponding elements in $G$ are adjacent or incident. Now we generalize this concept to $(1, \lambda)$-embedded graph, namely, a graph that can be embedded on a surface $S$ with Euler characteristic $\lambda$ so that each edge is crossed by at most one other edge. Actually, a $(1,2)$-embedded graph is a 1planar graph. It is shown in many papers such as [6] that $e(G) \leq 4 v(G)-8$ for every 1-planar graph $G$. Whereafter, to determine whether the number of edges in the class of $(1, \lambda)$-embedded graphs is linear or not linear in the number of vertices for every $\lambda \leq 2$ might be interesting.

In this paper, we first investigate some structures of $(1, \lambda)$-embedded graph $G$ in Section 2 and then give a relationship among the three parameters $e(G)$, $v(G)$ and $g(G)$ of $G$, which implies that every $(1, \lambda)$-embedded graph is 4-linear for any $\lambda \leq 2$. In Section 3, we will introduce a linear upper bound for the acyclic edge choice number of $(1, \lambda)$-embedded graphs with special given $\lambda$.

\section{The linearity of $(1, \lambda)$-embedded graphs}

Given a "good" graph $G$ (i.e., one for which all intersecting edges intersect in a single point and arise from four distinct vertices), the crossing number, 
denoted by $\operatorname{cr}(G)$, is the minimum possible number of crossings with which the graph can be drawn.

Let $G$ be a $(1, \lambda)$-embedded graph. In the following we always assume that $G$ has been embedded on a surface with Euler characteristic $\lambda$ so that each edge is crossed by at most one other edge and the number of crossings of $G$ in this embedding is minimum. Thus, $G$ has exactly $\operatorname{cr}(G)$ crossings. Sometimes we say such an embedding proper for convenience.

Theorem 2.1. Let $G$ be a $(1, \lambda)$-embedded graph. Then $\operatorname{cr}(G) \leq v(G)-\lambda$.

Proof. Suppose $G$ has been properly embedded on a surface with Euler characteristic $\lambda$. Then for each pair of edges $a b, c d$ that cross each other at a crossing point $s$, their end vertices are pairwise distinct. For each such pair, we add new edges $a c, c b, b d, d a$ (if it does not exist originally) to close $s$, then arbitrarily delete one edge $a b$ or $c d$ from $G$. Denote the resulting graph by $G^{*}$ and then we have $\operatorname{cr}\left(G^{*}\right)=0$. By Euler's formula $v\left(G^{*}\right)-e\left(G^{*}\right)+f\left(G^{*}\right)=\lambda$ and the well-known relation $\sum_{v \in V\left(G^{*}\right)} d_{V\left(G^{*}\right)}(v)=\sum_{f \in F\left(G^{*}\right)} d_{V\left(G^{*}\right)}(f)=2 e\left(G^{*}\right)$, $f\left(G^{*}\right) \leq 2 v\left(G^{*}\right)-2 \lambda$. Since each crossing point $s$ (note that $s$ is not a real vertex in $G$ ) lies on a common boundary of two faces of $G^{*}$ and each face of $G^{*}$ is incident with at most one crossing point (recall the definition of $\left.G^{*}\right)$, we deduce that $2 \operatorname{cr}(G) \leq f\left(G^{*}\right)$. Since $v(G)=v\left(G^{*}\right)$, we have $\operatorname{cr}(G) \leq \frac{f\left(G^{*}\right)}{2} \leq v\left(G^{*}\right)-\lambda=v(G)-\lambda$ in final.

Theorem 2.2. Let $G$ be a $(1, \lambda)$-embedded graph with girth at least $g$. Then $e(G) \leq \frac{2 g-2}{g-2}(v(G)-\lambda)$.

Proof. Suppose $G$ has been properly embedded on a surface with Euler characteristic $\lambda$. Now for each pair of edges $a b, c d$ that cross each other, we arbitrarily delete one from $G$. Let $G^{\prime}$ be the resulting graph. One can easily see that $\operatorname{cr}\left(G^{\prime}\right)=0$. By Euler's formula $v\left(G^{\prime}\right)-e\left(G^{\prime}\right)+f\left(G^{\prime}\right)=\lambda$ and the relations $v\left(G^{\prime}\right)=v(G), e\left(G^{\prime}\right)=e(G)-\operatorname{cr}(G)$, we have

$$
v(G)-e(G)+f\left(G^{\prime}\right)=v\left(G^{\prime}\right)-e\left(G^{\prime}\right)+f\left(G^{\prime}\right)-c r(G)=\lambda-\operatorname{cr}(G)
$$

and

$$
\begin{aligned}
\sum_{f \in F\left(G^{\prime}\right)} d_{G^{\prime}}(f)=2 e\left(G^{\prime}\right) & =2(e(G)-c r(G)) \\
& \geq g\left(G^{\prime}\right) f\left(G^{\prime}\right) \geq g(G) f\left(G^{\prime}\right) \geq g \cdot f\left(G^{\prime}\right)
\end{aligned}
$$

Now combine equations $(2.1)$ and $(2.2)$ together, we immediately have $e(G) \leq$ $\frac{g}{g-2}(v(G)-\lambda)+c r(G) \leq \frac{2 g-2}{g-2}(v(G)-\lambda)$ by Theorem 2.1 .

By Theorem 2.2, the following two corollaries are natural.

Corollary 2.3. Every $(1, \lambda)$-embedded graph is 4-linear for any $\lambda \leq 2$.

Corollary 2.4. Every triangle-free $(1, \lambda)$-embedded graph is 3-linear for any $\lambda \leq 2$. 


\section{Acyclic edge choosability of $(1, \lambda)$-embedded graphs}

In this section we mainly investigate the acyclic edge choosability of $(1, \lambda)$ embedded graphs with special given $\lambda$. In [7], Fiedorowicz et al. proved the following two results.

Theorem 3.1. If $G$ is a graph such that $e\left(G^{\prime}\right) \leq 2 v\left(G^{\prime}\right)-1$ for each $G^{\prime} \subseteq G$, then $\chi_{a}^{\prime}(G) \leq \Delta(G)+6$.

Theorem 3.2. If $G$ is a graph such that $e\left(G^{\prime}\right) \leq 3 v\left(G^{\prime}\right)-1$ for each $G^{\prime} \subseteq G$, then $\chi_{a}^{\prime}(G) \leq 2 \Delta(G)+29$.

In fact, these two theorems respectively imply that the acyclic edge chromatic number of 2-negative-linear graph $G$ is at most $\Delta(G)+6$ and that the acyclic edge chromatic number of 3-negative-linear graph $G$ is at most $2 \Delta(G)+29$

Note that every triangle-free $(1, \lambda)$-embedded graph is 3 -negative-linear for any $1 \leq \lambda \leq 2$ by Theorem 2.2. Hence the following corollary is trivial.

Corollary 3.3. Let $G$ be a triangle-free $(1, \lambda)$-embedded graph with $1 \leq \lambda \leq 2$. Then $\chi_{a}^{\prime}(G) \leq 2 \Delta(G)+29$.

The following main theorem in this section is dedicated to giving a linear upper bound for the acyclic edge choice number of 4-negative-linear graphs.

Theorem 3.4. If $G$ is a graph such that $e\left(G^{\prime}\right) \leq 4 v\left(G^{\prime}\right)-1$ for each $G^{\prime} \subseteq G$, then $\chi_{c}^{\prime}(G) \leq 3 \Delta(G)+70$.

As an immediately corollary of Theorems 2.2 and 3.4, we have the following result.

Corollary 3.5. Let $G$ be a $(1, \lambda)$-embedded graph with $1 \leq \lambda \leq 2$. Then $\chi_{c}^{\prime}(G) \leq 3 \Delta(G)+70$.

Before proving Theorem 3.4, we first show an useful structural lemma.

Lemma 3.6. Let $G$ be a graph such that $e(G) \leq 4 v(G)-1$ and $\delta(G) \geq 4$. Then at least one of the following configurations occurs in $G$ :

$(\mathcal{C} 1)$ a 4-vertex adjacent to a $19^{-}$-vertex;

$(\mathcal{C} 2)$ a 5 -vertex adjacent to two $19^{-}$-vertices;

(C3) a 6-vertex adjacent to four $19^{-}$-vertices;

$(\mathcal{C} 4)$ a 7 -vertex adjacent to six $19^{-}$-vertices;

$(\mathcal{C} 5)$ a vertex $v$ such that $20 \leq d(v) \leq 22$ and at least $d(v)-3$ of its neighbors are $7^{-}$-vertices;

$(\mathcal{C} 6)$ a vertex $v$ such that $23 \leq d(v) \leq 25$ and at least $d(v)-2$ of its neighbors are $7^{-}$-vertices;

$(\mathcal{C} 7)$ a vertex $v$ such that $26 \leq d(v) \leq 28$ and at least $d(v)-1$ of its neighbors are $7^{-}$-vertices;

$(\mathcal{C} 8)$ a vertex $v$ such that $29 \leq d(v) \leq 31$ and all its neighbors are $7^{-}$-vertices;

$(\mathcal{C} 9)$ a vertex $v$ such that at least $\bar{d}(v)-7$ of its neighbors are $7^{-}$-vertices and at least one of them is of degree 4. 
Proof. Suppose, to the contrary, that none of the nine configurations occurs in $G$. We assign to each vertex $v$ a charge $w(v)=d(v)-8$, then $\sum_{v \in V(G)} w(v)=$ $\sum_{v \in V(G)}(d(v)-8) \leq-2$. In the following, we will reassign a new charge denoted by $w^{\prime}(x)$ to each $x \in V(G)$ according to some discharging rules. Since our rules only move charges around, and do not affect the sum, we have

$$
\sum_{v \in V(G)} w^{\prime}(v)=\sum_{v \in V(G)} w(v) \leq-2 .
$$

We next show that $w^{\prime}(v) \geq 0$ for each $v \in V(G)$, which leads to a desired contradiction. We say a vertex big (resp. small) if it is a $20^{+}$-vertex (resp. $7^{-}$vertex). The discharging rules are defined as follows.

$(\mathcal{R} 1)$ Each big vertex gives 1 to each adjacent 4 -vertex.

$(\mathcal{R} 2)$ Each big vertex gives $\frac{3}{4}$ to each adjacent vertex of degree between 5 and 7 .

Let $v$ be a 4 -vertex. Since $(\mathcal{C} 1)$ does not occur, $v$ is adjacent to four big vertices. So $v$ totally receives 4 by $(\mathcal{R} 1)$. This implies that $w^{\prime}(v)=w(v)+4=$ $d(v)-4=0$. Similarly, we can also prove the nonnegativity of $w^{\prime}(v)$ if $v$ is a $k$-vertex with $5 \leq k \leq 7$. Let $v$ be a $k$-vertex with $8 \leq k \leq 19$. Since $v$ is not involved in the discharging rules, $w^{\prime}(v)=w(v)=d(v)-8 \geq 0$. Let $v$ be a $k$-vertex with $20 \leq k \leq 22$. If $v$ is adjacent to a 4 -vertex, then $v$ is adjacent to at most $d(v)-8$ small vertices since $(\mathcal{C} 9)$ does not occur. Since $v$ sends each small vertex at most 1 by $(\mathcal{R} 1)$ and $(\mathcal{R} 2), w^{\prime}(v) \geq w(v)-(d(v)-8)=0$. If $v$ is adjacent to no 4 -vertices, then $v$ sends each small vertex $\frac{3}{4}$ by $(\mathcal{R} 2)$. Since $(\mathcal{C} 5)$ does not occur either, $v$ is adjacent at most $d(v)-4$ small vertices. So $w^{\prime}(v) \geq w(v)-\frac{3}{4}(d(v)-4)=\frac{1}{4}(d(v)-20) \geq 0$. By similar arguments as above, we can also respectively show the nonnegativity of $w^{\prime}(v)$ if $v$ is a $k$-vertex with $k \geq 23$.

Proof of Theorem 3.4. Let $K$ stands for $3 \Delta(G)+70$. We prove the theorem by contradiction. Let $G$ be a counterexample to the theorem with the number of edges as small as possible. So there exists a list assignment $L$ of $K$ colors such that $G$ is not acyclicly edge- $L$-choosable. For each coloring $c$ of $G$, we define $c(u v)$ to be the color of edge $u v$ and set $C(u)=\{c(u v) \mid u v \in E(G)\}$ for each vertex $u$. For $W \subseteq V(G)$, set $C(W)=\bigcup_{w \in W} C(w)$. If $u v \in E(G)$, we let $W_{G}(v, u)$ stands for the set of neighbors $w$ of $v$ in $G$ such that $c(v w) \in C(u)$. Now, we first prove that $\delta(G) \geq 4$.

Suppose that there is a 3 -vertex $v \in V(G)$. Denote the three neighbors of $v$ by $x, y$ and $z$. Then by the minimality of $G$, the graph $H=G-u x$ is acyclicly edge- $L$-choosable. Let $c$ be an acyclic edge coloring of $H$. We can extend $c$ to $u v$ by defining a list of available colors for $u v$ as follows:

$$
A(u v)=L(u v) \backslash(C(x) \cup C(y) \cup C(z)) .
$$

Since $|C(x)| \leq \Delta(G)-1,|C(y)| \leq \Delta(G)$ and $|C(z)| \leq \Delta(G)$, we have $|A(u v)| \geq$ $K-3 \Delta(G)+1>0$. So we can color $u v$ by a color in $A(u v) \subseteq L(u v)$, a 
contradiction. Similarly, one can also prove the absences of 1-vertices and 2vertices in $G$. Hence $\delta(G) \geq 4$. Then by Lemma 3.6, $G$ contains at least one of the configurations $(\mathcal{C} 1)-(\mathcal{C} 9)$. In the following, we only show that if one of the configurations $(\mathcal{C} 4),(\mathcal{C} 5)$ and $(\mathcal{C} 9)$ appears, then we would get a contradiction. That is because the proofs are similar and easier for another six cases.

Configuration $(\mathcal{C} 4)$ : Suppose that there is a 7 -vertex $v$ that is adjacent to six $19^{-}$-vertices, say $x_{1}, x_{2}, \ldots, x_{6}$. Denote another one neighbor of $v$ by $x_{7}$. Then by the minimality of $G$, the graph $H=G-v x_{7}$ is acyclicly edge- $L$-choosable. Let $c$ be an acyclic edge coloring of $H$. Suppose $c\left(v x_{j}\right) \notin C\left(x_{7}\right)$ for some $1 \leq j \leq 6$. Then we can extend $c$ to $v x_{7}$ by defining a list of available colors for $v x_{7}$ as follows:

$$
A\left(v x_{7}\right)=L\left(v x_{7}\right) \backslash \bigcup_{1 \leq i \neq j \leq 7} C\left(x_{i}\right) .
$$

Since $\left|C\left(x_{i}\right)\right| \leq \min \{19, \Delta(G)\}$ for every $1 \leq i \leq 6$ and $\left|C\left(x_{7}\right)\right| \leq \Delta(G)-1$, we have $\left|A\left(v x_{7}\right)\right| \geq K-\min \{\Delta(G)+94,6 \Delta(G)-1\}=\max \{2 \Delta(G)-24,-3 \Delta(G)+$ $69\}>0$. So we can color $v x_{7}$ by a color in $A\left(v x_{7}\right) \subseteq L\left(v x_{7}\right)$, a contradiction. Thus we shall assume that $c\left(v x_{j}\right) \in C\left(x_{7}\right)$ for every $1 \leq j \leq 6$. This implies that $\left|\bigcup_{1 \leq i \leq 7} C\left(x_{i}\right)\right| \leq \Delta(G)-1+6 \times \min \{19, \Delta(G)\}-6=\min \{\Delta(G)+$ $107,7 \Delta(G)-7\}$. Now we extend $c$ to $v x_{7}$ by defining a list of available colors for $v x_{7}$ as follows:

$$
A\left(v x_{7}\right)=L\left(v x_{7}\right) \backslash \bigcup_{1 \leq i \leq 7} C\left(x_{i}\right) .
$$

Note that $\left|A\left(v x_{7}\right)\right| \geq K-\min \{\Delta(G)+107,7 \Delta(G)-7\}=\max \{2 \Delta(G)-$ $37,-4 \Delta(G)+77\}>0$. So we can again color $v x_{7}$ by a color in $A\left(v x_{7}\right) \subseteq L\left(v x_{7}\right)$, also a contradiction.

Configuration $(\mathcal{C} 5)$ : If there is a vertex $v$ such that $20 \leq d(v) \leq 22$ and at least $d(v)-3$ of its neighbors are $7^{-}$-vertices. Without loss of generality, we assume that $d(v)=22$ and that $v$ have nineteen $7^{-}$-neighbors. Denote another three neighbors of $v$ by $x, y$ and $z$. Choose one $7^{-}$-neighbor, say $u$, of $v$. Without loss of generality, we assume that $d(u)=7$. Then by the minimality of $G$, the graph $H=G-u v$ is acyclicly edge- $L$-choosable. Let $c$ be an acyclic edge coloring of $H$. Then we can extend $c$ to $u v$ by defining a list of available colors for $u v$ as follows:

$$
A(u v)=L(u v) \backslash\left\{C(u) \cup C(v) \cup C(x) \cup C(y) \cup C(z) \cup C\left(W_{H}(v, u)\right\} .\right.
$$

Since $c$ is an acyclic (and thus it is proper), $\left|W_{H}(v, u)\right| \leq d(u)-1=6$. Since $|C(x)| \leq \Delta(G),|C(y)| \leq \Delta(G),|C(z)| \leq \Delta(G)$ and $|C(w)| \leq 7$ for each $w \in W_{H}(v, u)$, we have $|A(u v)| \leq K-(3 \Delta(G)+6+6 \times 6+21-9)>0$. So we can color $u v$ by a color in $A(u v) \subseteq L(u v)$, a contradiction.

Configuration $(\mathcal{C} 9)$ : If there is a vertex $v$ such that at least $d(v)-7$ of its neighbors are $7^{-}$-vertices and at least one of them is of degree 4 , say $u$. Denote another three neighbors of $u$ by $x, y$ and $z$. Let $C_{1}=\{c(v w) \mid w \in$ $N_{H}(v)$ and $\left.d_{H}(w)>7\right\}$ and $C_{2}=\left\{c(v w) \mid w \in N_{H}(v)\right.$ and $\left.d_{H}(w) \leq 7\right\}$. Then 
$\left|C_{1}\right| \leq 7$. By the minimality of $G$, the graph $H=G-u v$ is acyclicly edge$L$-choosable. Let $c$ be an acyclic edge coloring of $H$. Suppose $C(u) \cap C_{1} \neq \emptyset$. Without loss of generality, we assume that $c(u x) \in C_{1}$. Now we erase the color of the edge $u x$ from $c$ and recolor it from the list defined as follows:

$$
A(u x)=L(u x) \backslash\left\{C(x) \cup C(y) \cup C(z) \cup C_{1}\right\} .
$$

Since $|C(x)| \leq \Delta(G),|C(y)| \leq \Delta(G),|C(z)| \leq \Delta(G)$ and $\left|C_{1}\right| \leq 7$, we have $|A(u x)| \geq K-(3 \Delta(G)+7)>0$. Note that $A(u x)$ is just a sub-list of the original list given at the beginning of the proof and the new color of $u x$ preserves the acyclicity of the coloring of $H$. So we can assume that $C(u) \cap C_{1}=\emptyset$. In this case, we can extend $c$ to the edge $u v$ by defining a list of available colors for $u v$ as follows:

$$
A(u v)=L(u v) \backslash\left\{C(u) \cup C_{1} \cup C_{2} \cup C\left(W_{H}(v, u)\right)\right\} .
$$

Since $C(u) \cap C_{1}=\emptyset$, we have $c(v w) \in C_{2}$ for each $w \in W_{H}(v, u)$ and thus $\left|C\left(W_{H}(v, u)\right)\right| \leq 7 d_{H}(u)=21$. Since $\left|C_{1} \cup C_{2}\right|=d_{H}(v) \leq \Delta(G)-1$ and $|C(u)|=3$, we have $|A(u v)| \geq K-(\Delta(G)+23)>0$. So we can color $u v$ by a color in $A(u v) \subseteq L(u v)$. This contradiction completes the proof of Theorem 3.4 .

\section{References}

[1] N. Alon, C. J. H. McDiarmid, and B. A. Reed, Acyclic coloring of graphs, Random Structures Algorithms 2 (1991), no. 3, 277-288.

[2] N. Alon, B. Sudakov, and A. Zaks, Acyclic edge-colorings of graphs, J. Graph Theory 37 (2001), no. 3, 157-167.

[3] J. A. Bondy and U. S. R. Murty, Graph Theory with Applications, North-Holland, New York, 1976.

[4] T. F. Coleman and J. Cai, The cyclic coloring problem and estimation of sparse Hessian matrices, SIAM J. Algebraic Discrete Methods 7 (1986), no. 2, 221-235.

[5] T. F. Coleman and J. J. Moré, Estimation of sparse Hessian matrices and graph coloring problems, Math. Programming 28 (1984), no. 3, 243-270.

[6] I. Fabrici and T. Madaras, The structure of 1-planar graphs, Discrete Math. 307 (2007), no. 7-8, 854-865.

[7] A. Fiedorowicz, M. Halszczak, and N. Narayanan, About acyclic edge colourings of planar graphs, Inform. Process. Lett. 108 (2008), no. 6 412-417.

[8] B. Grünbaum, Acyclic colorings of planar graphs, Israel J. Math. 14 (1973), 390-408.

[9] M. Molloy and B. Reed, Further algorithmic aspects of the local lemma, In: it Proceedings of the 30th Annual ACM Symposium on Theory of Computing (1998), pp. 524-529.

[10] S. Ndreca, A. Procacci, and B. Scoppola, Improved bounds on coloring of graphs, available online at http://arxiv.org/pdf/1005.1875v1.

[11] G. Ringel, Ein sechsfarbenproblem auf der Kugel, Abh. Math. Sem. Univ. Hamburg 29 (1965), 107-117.

XIN ZHANG

SCHOOL OF Mathematics

SHANDONG UNIVERSITY

JiNAN 250100, P. R. CHINA

E-mail address: sdu.zhang@yahoo.com.cn 
Guizhen LiU

School of Mathematics

SHANDONG UNIVERSITY

JinAN 250100, P. R. CHINA

E-mail address: gzliu@sdu.edu.cn

JIAN-LIANG WU

School of Mathematics

SHANDONG UNIVERSITY

JinAN 250100, P. R. CHINA

E-mail address: jlwu@sdu.edu.cn 